\title{
EFFICIENCY OF AN EXPERIMENTAL MEROPENEM EYE DROP FORMULATION IN SELECTED CASES OF CANINE AND FELINE INFECTIOUS CONJUNCTIVITIS
}

\section{FABIANO MONTIANI FERREIRA ${ }^{1}$; CLÁUDIA DOTTI ${ }^{2}$}

${ }^{1}$ Professor of Internal Medicine, Departament of Veterinary Medicine, Federal University of Paraná. ${ }^{2}$ Senior Veterinary

Student, Federal University of Paraná.

Meropenem is the newest beta-lactam antibiotic of the carbapenem family. According to PAPICH (1998), the use of meropenem has not been reported in veterinary medicine. This drug has the broadest antimicrobial spectrum known by man. An eye drop solution was developed in a specialized laboratory (Ophthalmos@- São Paulo) using the drug salt (Meronem-Zeneca ${ }^{\circledR}$ ). The final solution had a concentration of $5 \mathrm{mg} / \mathrm{ml}$ and the vehicle used was $\mathrm{NaCl} 0,9 \%$ solution. There was no addition of preservatives and the drug bottle had to be kept inside of a refrigerator after opened, even though, shelf life was very short, just 48 hours. Six cases of infectious conjunctivitis were chosen to be treated with this experimental formulation. Four dogs and two cats were selected. All patients were selected using five criteria: presence of conjunctivitis, Schirmer tear test values, conjunctival cytology, previous use of another antibiotic and bacterial culture results. The patients selected have had normal Schirmer test values, absence of intracellular organisms on conjunctival cells and did not received any other topic or systemic antimicrobial drug, before or during the treatment. Ocular secretions were collected using a cotton swab. All samples were taken to the laboratory on sterile tube containing agar gel. Culture results revealed four cases in which the infection was caused by coagulase negative Staphylococcus spp. (three dogs and one cat), in one case the infection was caused by Streptococcus pyogenes (dog) and finally one infection was caused by Pasteurella multocida (cat). The treatment with the drug solution consisted of one drop on the affected eye every six hours (QID) for ten days. All cases have responded extremely well to the eye drop treatment. Additionally, all patients did not show intolerance or any sign of pain when the drug was administered. Four infections resolved after four days, one resolved after five days. The P. multocida infection case resolved only after six days of treatment. Results show that this new drug has a very promising future in veterinary medicine, depending much on its future price on the market. Currently, we are developing Kirby-Bauer sensitivity discs with meropenem in order to test the in vitro spectrum of action more intensively. This is the first report of the use of this drug in veterinary medicine. 